Check for updates

Cite this: Chem. Commun., 2020, 56, 11665

Received 20th May 2020

Accepted 25th August 2020

DOI: $10.1039 / \mathrm{d} 0 \mathrm{cc} 03612 \mathrm{a}$

rsc.li/chemcomm

\section{A quantitative assay to study the lipid selectivity of membrane-associated systems using solution NMR $\dagger$}

\author{
Encarnacion Medina-Carmona, ${ }^{a b}$ Lorena Varela, ${ }^{a}$ Alex C. Hendry, ${ }^{a}$ \\ Gary S. Thompson, $\mathbb{D}^{a}$ Lisa J. White, ${ }^{c}$ Jessica E. Boles, ${ }^{c}$ Jennifer R. Hiscock (D) ${ }^{c}$ \\ and Jose L. Ortega-Roldan (D) *a
}

The activity of membrane proteins and compounds that interact with the membrane is modulated by the surrounding lipid composition. However, there are no simple methods that determine the composition of these annular phospholipids in eukaryotic systems. Herein, we describe a simple methodology that enables the identification and quantification of the lipid composition around membrane-associated compounds using SMA-nanodiscs and routine ${ }^{1} \mathrm{H}-{ }^{31} \mathrm{P}$ NMR.

The structure and function of membrane proteins, synthetic channels and any membrane-associated compound can be influenced by the lipid environment, yet the precise composition of lipids surrounding them is often difficult to discern. ${ }^{1,2}$ Additionally, understanding lipid specificity in biological membranes is crucial for the understanding of the modulation of membrane protein activity in cells as well as for the design and development of chemical molecules that interact with biological membranes such as antimicrobials, carriers, or synthetic channels. ${ }^{3,4}$

Two different classes of lipids can be identified around membrane-embedded systems: (1) non-annular lipids are those tightly bound to cavities in the hydrophobic regions of the protein (or compound) and are non-exchangeable. Non-annular lipids can be resistant to membrane solubilisation by harsh treatment with detergent micelles and, in the case of integral membrane proteins, could be subsequently detected by co-crystallisation or native mass spectrometry. ${ }^{5,6}$ (2) Annular lipids constitute the first layer of lipids surrounding the membrane-bound system and have restricted mobility compared to bulk lipids, but may exchange with the bulk lipids of

\footnotetext{
${ }^{a}$ School of Biosciences, University of Kent, Canterbury, CT2 7NJ, UK.

E-mail: J.L.Ortega-Roldan@kent.ac.uk; Tel: +44 (0)1227 824730

${ }^{b}$ Departamento de Quimica-Fisica, Facultad de Ciencias, Universidad de Granada, Granada 18010, Spain

${ }^{c}$ School of Physical Sciences, University of Kent, Canterbury, CT2 7NJ, UK

$\dagger$ Electronic supplementary information (ESI) available: This includes experimental details and NMR spectroscopy data. See DOI: 10.1039/d0cc03612a
}

the membrane. The detection of annular lipids is not possible for membrane-bound systems extracted in detergent micelles, and their influence is commonly assayed using reconstituted vesicles of different lipid compositions. ${ }^{6,7}$ It is generally assumed that membrane-bound systems will be surrounded by those lipids that render their maximal activity. ${ }^{5}$ However, this approach does not enable the study of lipid selectivity by membrane proteins or membrane-interacting compounds in their host biological membranes, and is not sensitive to alterations in the specific lipid composition of the annular and non-annular lipids surrounding membrane-bound compounds.

The development of native styrene-maleic acid copolymer (SMA) nanodiscs has enabled the extraction of membraneassociated compounds from native membranes. ${ }^{8,9}$ SMA nanodiscs have been shown to maintain many desirable physical properties of the membrane bilayer, such as lipid phase transition temperatures and lipid bilayer thickness. ${ }^{10,11}$ Recently, new families of SMA have also been developed expanding the range of conditions in which this type of membrane mimetic can be utilised. ${ }^{12}$ In addition, the use of nanodiscs has been shown to be a useful tool for studying the chemical interactions between small molecules and the phospholipid bilayer, which is of great importance for the development of novel pharmaceuticals, drug delivery systems, synthetic membrane transporters or ion channel technologies. ${ }^{13}$ The combination of SMA solubilisation with thin layer chromatography and mass spectrometry has allowed the identification of lipids co-extracted with different membrane proteins, in bacterial systems and simple lipid mixtures. ${ }^{14-16}$ However, such approaches are not easily applicable to eukaryotic membranes due to the complexity of their phospholipid composition, also they do not allow for lipid quantification without the addition of non-natural lipid internal standards for each lipid analysed.

Herein, we present an easily accessible solution state NMR methodology that allows the identification and quantification of the specific phospholipid headgroup composition around membrane-associated systems in their natural lipid environment. 


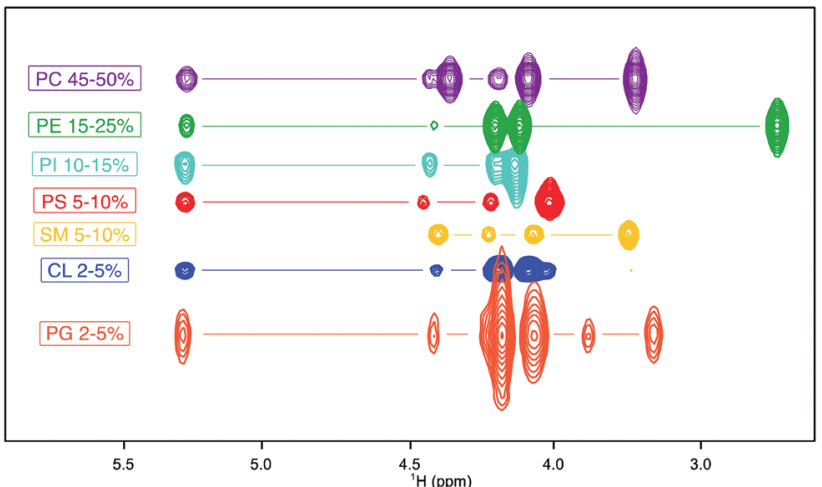

Fig. 1 Overlay of ${ }^{1} \mathrm{H}$ fingerprints of standard mammalian phospholipids from ${ }^{1} \mathrm{H}-{ }^{31} \mathrm{P} H S Q C$ spectra arranged by abundance ${ }^{1}$ from the most abundant (top) to the least (bottom). PC (purple), PE (green), PI (cyan), PS (red), SM (yellow), CL (dark blue) and PG (orange). The spectra were collected in in $75 \% \mathrm{CDCl}_{3}, 25 \% \mathrm{MeOD}$, and $0.05 \%$ TMS.

This can be applied to both membrane-associated and transmembrane proteins, synthetic channels, or generally to any compounds tightly associated to any phospholipid membrane.

This methodology combines an extraction step using SMAnanodiscs with a simple ${ }^{1} \mathrm{H}^{-31} \mathrm{P}$ NMR experiment that allows both the identification and quantification of lipid headgroups present in the mixture (including annular lipids). Phosphorus NMR is routinely used for determination and quality control of phospholipids. However, ${ }^{31} \mathrm{P}$ NMR experiments are insensitive and display a narrow dispersion of the lipid NMR resonances. To alleviate both problems, we turned to a proton start ${ }^{1} \mathrm{H}^{-31} \mathrm{P}$ HSQC (ESI $\dagger$ ).

Firstly, we collected ${ }^{1} \mathrm{H}^{31} \mathrm{P}$ HSQC spectra of the most common lipids that form the biological membranes (Fig. S1, ESI $\dagger$ ) and which have at least one phosphorus molecule in their headgroup (dimyristoyl phosphatidyl choline (PC), palmitoyloleoyl phosphatidyl ethanolamine (PE), dipalmitoyl phosphatidyl serine (PS), soy phosphatidyl inositol (PI), brain sphingomyelin (SM), egg phosphatidyl glycerol (PG) and cardiolipin (CL) (Fig. S2-S8 respectively, ESI $\dagger$ ). Each lipid shows a unique ${ }^{1} \mathrm{H}$ resonance pattern irrespective of its acyl chain (Fig. 1) that allows for the correct headgroup identification.

Phosphorus chemical shifts have already been used for phospholipid identification, ${ }^{17-19}$ however we detected a linear dependence of ${ }^{31} \mathrm{P}$ chemical shifts of each individual lipid with the total lipid concentration. This is likely due to crowding effects which prevents lipid identification based solely on ${ }^{31} \mathrm{P}$ chemical shifts in complex lipid mixtures (Fig. S9-S12, ESI $\dagger$ ). The combination of both ${ }^{1} \mathrm{H}$ and ${ }^{31} \mathrm{P}$ resonances provide unique patterns that enable the identification of all the lipids in our library by comparison with the ${ }^{1} \mathrm{H}$ resonances and the expected ${ }^{31} \mathrm{P}$ chemical shifts in complex phospholipid mixtures, such as eukaryotic membranes (Fig. S13, ESI $\dagger$ ).

Relative lipid quantification was then carried out using the ${ }^{31} \mathrm{P}$ 1D skyline projection of the ${ }^{1} \mathrm{H}^{-31} \mathrm{P}$ HSQC spectra, where every point has the highest intensity of all points of the corresponding orthogonal ${ }^{1} \mathrm{H}$ traces in the $2 \mathrm{D}$ spectrum, used due to its high sensitivity and ease of use. Absolute areas were

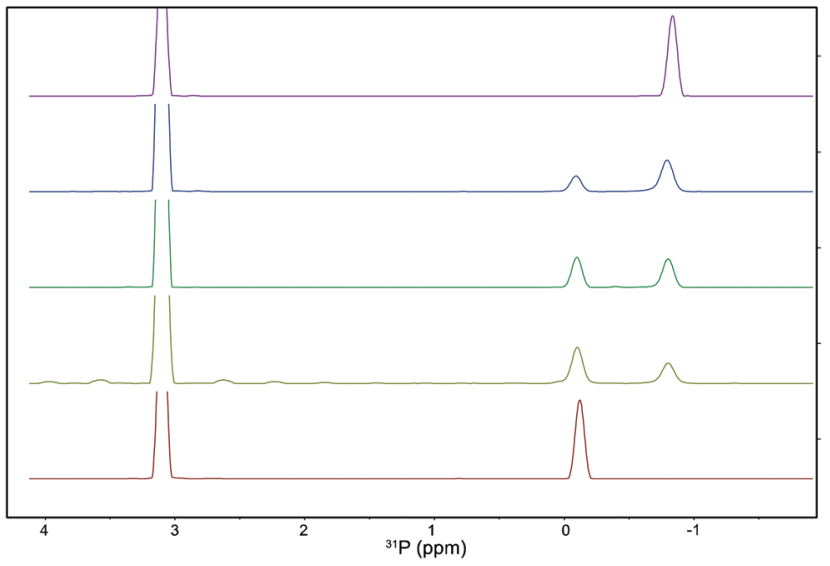

Fig. $2{ }^{31} \mathrm{P} 1 \mathrm{D}$ skyline projections from ${ }^{1} \mathrm{H}-{ }^{31} \mathrm{P}$ HSQC spectra of samples containing mixtures PC: PE in the ratios $100: 0 \%$ (top), $72: 28 \%, 50: 50 \%$, $33: 66 \%$, and $0: 100 \%$ (bottom).

calculated and normalised against the integral of trimethyl phosphate (TMP) as an internal standard, enabling the quantification of the relative proportions of each phospholipid component in the mixture. To validate this method, five samples with known concentrations of PC and PE lipids were prepared and the relative concentrations of both phospholipids were determined (Fig. 2). Linear fitting of the data showed excellent accuracy, with a slope of 0.9966 and a correlation coefficient $(R)$ of 0.9999 (Fig. S20, ESI $\dagger$ ). We verified our NMR-based identification and quantification with a commercial Escherichia coli (E. coli) lipid extract and with eukaryotic lipid mixtures extracted from Colo-680N cell lines (Fig. S13 and S14, ESI†). In all cases, our calculated compositions correlate well with experimentally derived compositions. ${ }^{1}$

To study the lipid selectivity of membrane-associated systems, we devised a strategy where purified proteins or membrane-associated compounds are incorporated into lipid vesicles from their host cell type or containing lipid mixtures of a desired composition and extracted using SMA. For SMA extraction, the vesicles are incubated with $2 \%$ SMA, dialysed and purified using a native chromatography technique. Size exclusion chromatography was used to separate empty or loaded SMA-nanodiscs and any free SMA (Fig. S15, ESI $\dagger$ ). Moreover, we observed that in the presence of an excess of membrane-associated compound the SMA nanodiscs eluted at a lower retention volume, suggesting that they are predominantly loaded. The relevant empty or loaded SMA nanodisc fractions are then subjected to the Folch lipid extraction method, ${ }^{20}$ with the lipids then dried under a nitrogen stream. The dried lipids were then resuspended in a $1: 1 \mathrm{CDCl}_{3}: \mathrm{MeOD}-$ $d_{4}$ solvent mixture, with the reference compound TMP. $\mathrm{A}^{1} \mathrm{H}^{3}{ }^{31} \mathrm{P}-\mathrm{HSQC}$ spectrum was then collected. To rule out any influence of SMAs on the lipid composition, we compared the lipid composition of empty SMA nanodiscs against the original vesicles. No significant differences were observed, confirming the suitability of our approach (Fig. S16, ESI $\dagger$ ).

For this proof of principle study, we studied the lipid composition surrounding a membrane-associated protein, the 

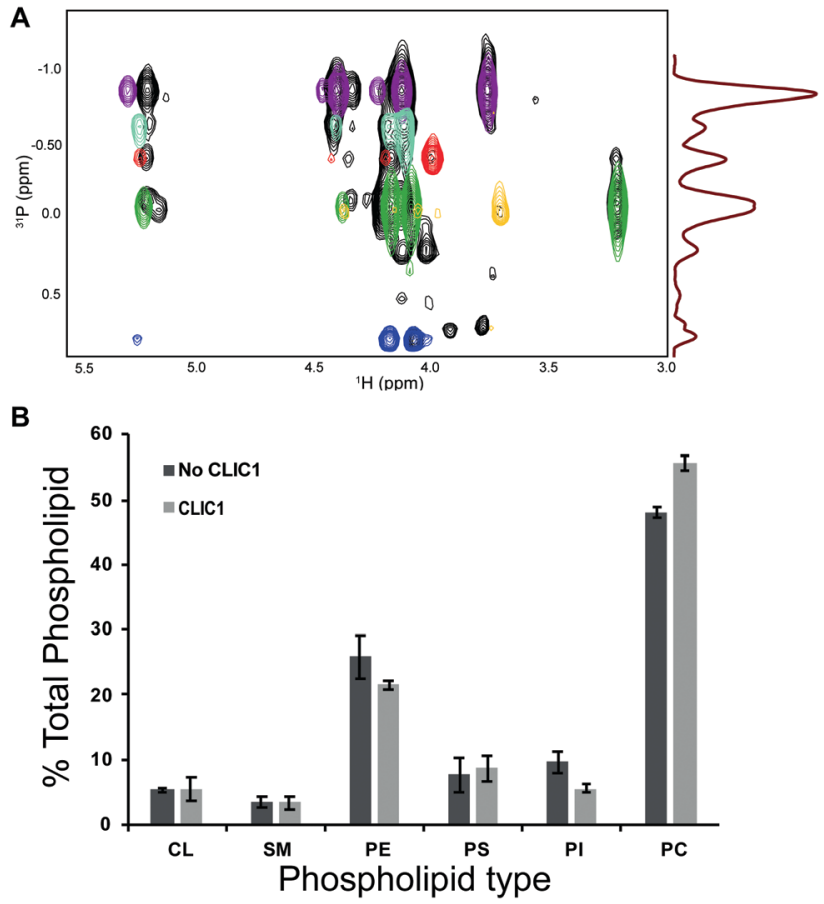

Fig. 3 (A) Overlay of ${ }^{31} \mathrm{P}$ HSQC spectra of phospholipids extracted from CLIC1-containing SMA nanodisCs formed with COLO-680N cells lipid extract (black) and the reference lipids overlaid based on the ${ }^{1} \mathrm{H}$ patterns $(\mathrm{PC}=$ purple, $\mathrm{PE}=$ green, $\mathrm{PI}=$ light blue, $\mathrm{PS}=$ red, $\mathrm{SM}=$ yellow , cardiolipin = dark blue). (B) Differences in total phospholipid composition between samples with and without CLIC1 $(n=3)$.

chloride intracellular channel-1 (CLIC1) and a membraneassociated compound, a representative compound from a novel class of supramolecular self-associating amphiphile (SSA) with antimicrobial activity. ${ }^{21}$ CLIC1, a member of the CLIC family of proteins, is expressed as a soluble protein in human cells, but inserts in the membrane through interaction with divalent cations, forming a chloride channel. ${ }^{22-24}$ This chloride channel form is upregulated in different types of cancers including glioblastoma and promotes tumour invasiveness and metastasis. $^{25,26}$ The chloride efflux activity has been shown to be modulated by the lipid composition, with maximum chloride efflux in soybean lipid extract, enriched with PC and PE lipids. ${ }^{23}$ A high chloride efflux activity of CLIC1 in the plasma membrane has been associated with disease states, and therefore we questioned if the cells would tune CLIC1 to give maximal activity or would rather regulate it to lower levels. ${ }^{27}$

We assayed the lipid selectivity of CLIC1 in eukaryotic membranes. The vesicles formed with lipids extracted from eukaryotic membranes were incubated with purified CLIC1 or buffer, and subsequently extracted with SMAs. The empty and protein containing SMA-nanodiscs were purified as described above. The lipids contained in the discs were extracted and assayed by ${ }^{1} \mathrm{H}^{3}{ }^{31} \mathrm{P}$ HSQC NMR. The analysis of these samples showed differences in the total distribution of lipids due to the presence of CLIC1 (Fig. 3). These results provide an indication of the lipid selectivity of CLIC1 close to physiological conditions, with a $7.5 \%$ change in PC and $4 \%$ change in PE and PI,
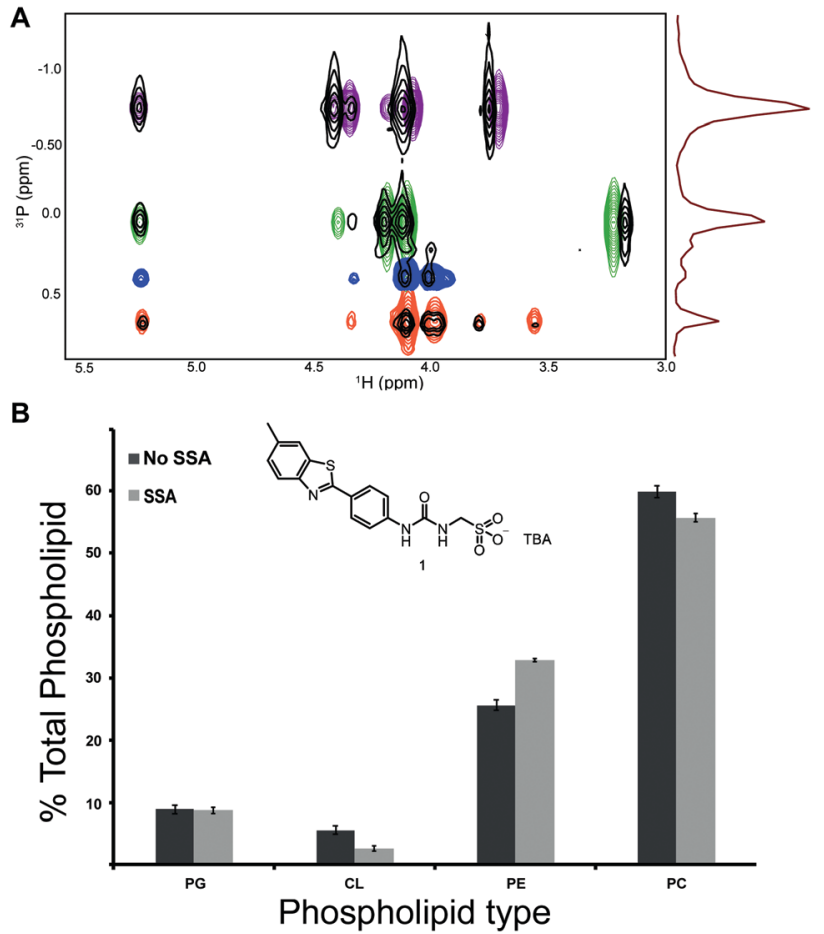

Fig. 4 (A) Overlay of ${ }^{31} \mathrm{P}$ HSQC spectra of phospholipids extracted from SSA compound 1-containing SMA nanodiscs formed with $E$. coli lipid extract mixed with DMPC (2:3) (black) and the reference lipids overlaid based on the ${ }^{1} \mathrm{H}$ patterns $(\mathrm{PC}=$ purple, $\mathrm{PE}=$ green, $\mathrm{PI}=$ light blue, $\mathrm{PS}=$ red, $\mathrm{SM}=$ yellow, cardiolipin = dark blue). (B) Differences in total phospholipid composition between samples with and without SSA (ESI $\dagger)(n=3)$. The chemical structure of SSA compound 1 is included. TBA = tetrabutylammonium.

which supports the idea that CLIC1 is not regulated to its maximum activity despite its lipid selectivity. Furthermore, the sensitivity of the method allows the detection of a small preference for certain types of phospholipids by CLIC1.

We then applied this novel assay to ascertain the selectivity of non-protein compounds for different types of phospholipid headgroups. We previously showed a compound from the antimicrobial agent SSA library to have higher affinity towards SMA nanodiscs derived from $E$. coli phospholipids over those derived from DMPC-mimicking a general human cell surface. ${ }^{13}$ Following an analogous approach to that described previously, but instead incubating this SSA with a mixture of E. coli total lipid extract and DMPC in a $2: 3$ ratio, followed by SMA extraction. Phospholipid headgroup analysis indicated an enrichment of $6 \%$ in PE lipids at the cost of a decrease in the content of DMPC (Fig. 4), in agreement with previous results. It is hypothesised that quantifying these molecular level interactions will enable the tuning of this SSA technology towards bacterial, over eukaryotic cell membranes, reducing the potential for these SSAs to illicit toxic effects when supplied to a system in vivo.

In summary, we present a simple methodology that enables, for the first time, the identification and quantification of small differences in the phospholipid composition of any type of biological membrane, including complex eukaryotic phospholipid mixtures, induced by the lipid selectivity of either 
membrane-associated proteins or synthetic compounds. Due to the sensitivity of the ${ }^{1} \mathrm{H}-{ }^{31} \mathrm{P}-\mathrm{HSQC}$, sub-milligram amounts of proteins or chemical compounds and lipids are sufficient. This method also allows the identification of other types of phospholipids, provided that the ${ }^{1} \mathrm{H}^{-31} \mathrm{P}$ resonances are known, although is insensitive to different acyl chains or to lipids not containing phosphorus groups. Furthermore, this strategy can be an excellent resource for the design and development of chemical molecules that interact with biological membranes such as antimicrobials, carriers, or synthetic channels.

The authors would like to acknowledge Dr Timothy Knowles (University of Birmingham) for his assistance with this work. The authors would also like to thank the following organisations for funding this work: the Wellcome Trust (207743/Z/17/Z), the Royal Society (RGS $\backslash$ R1 $\backslash 191414$ ) and Public Health England (JEB PhD studentship).

\section{Conflicts of interest}

There are no conflicts to declare.

\section{Notes and references}

1 P. V. Escribá, X. Busquets, J. Inokuchi, G. Balogh, Z. Török, I. Horváth, J. L. Harwood and L. Vígh, Prog. Lipid Res., 2015, 59, 38-53.

2 A. Laganowsky, E. Reading, T. M. Allison, M. B. Ulmschneider, M. T. Degiacomi, A. J. Baldwin and C. V. Robinson, Nature, 2014, 510, 172-175.

3 P. Maturana, M. Martinez, M. E. Noguera, N. C. Santos, E. A. Disalvo, L. Semorile, P. C. Maffia and A. Hollmann, Colloids Surf., B, 2017, 153, 152-159.

4 M. Zhang, P.-P. Zhu, P. Xin, W. Si, Z.-T. Li and J.-L. Hou, Angew. Chem., Int. Ed., 2017, 56, 2999-3003.

5 C. Schölz, D. Parcej, C. S. Ejsing, H. Robenek, I. L. Urbatsch and R. Tampé, J. Biol. Chem., 2011, 286, 13346-13356.

6 J. A. Poveda, A. M. Giudici, M. L. Renart, M. L. Molina, E. Montoya, A. Fernández-Carvajal, G. Fernández-Ballester, J. A. Encinar and J. M. González-Ros, Biochim. Biophys. Acta, Biomembr., 1838, 2014, 1560-1567.

7 A. G. Lee, Biochim. Biophys. Acta, Biomembr., 2004, 1666, 62-87.
8 K. S. Simon, N. L. Pollock and S. C. Lee, Biochem. Soc. Trans., 2018, 46, 1495-1504.

9 J. M. Dörr, S. Scheidelaar, M. C. Koorengevel, J. J. Dominguez, M. Schäfer, C. A. van Walree and J. A. Killian, Eur. Biophys. J., 2016, 45, 3-21.

10 P. Angelisová, O. Ballek, J. Sýkora, O. Benada, T. Čajka, J. Pokorná, D. Pinkas and V. Hořejší, Biochim. Biophys. Acta, Biomembr., 1861, 2019, 130-141.

11 M. Jamshad, V. Grimard, I. Idini, T. J. Knowles, M. R. Dowle, N. Schofield, P. Sridhar, Y. Lin, R. Finka, M. Wheatley, O. R. T. Thomas, R. E. Palmer, M. Overduin, C. Govaerts, J.-M. Ruysschaert, K. J. Edler and T. R. Dafforn, Nano Res., 2014, 8, 774-789.

12 T. Ravula, N. Z. Hardin, S. K. Ramadugu, S. J. Cox and A. Ramamoorthy, Angew. Chem., Int. Ed., 2018, 57, 1342-1345.

13 G. Townshend, G. S. Thompson, L. J. White, J. R. Hiscock and J. L. Ortega-Roldan, Chem. Commun., 2020, 56, 4015-4018.

14 V. Schmidt, M. Sidore, C. Bechara, J.-P. Duneau and J. N. Sturgis, Biochim. Biophys. Acta, Biomembr., 2019, 1861, 431-440.

15 A. C. K. Teo, S. C. Lee, N. L. Pollock, Z. Stroud, S. Hall, A. Thakker, A. R. Pitt, T. R. Dafforn, C. M. Spickett and D. I. Roper, Sci. Rep., 2019, 9, 1-10.

16 I. Prabudiansyah, I. Kusters, A. Caforio and A. J. M. Driessen, Biochim. Biophys. Acta, Biomembr., 2015, 1848, 2050-2056.

17 B. Gouilleux, N. V. Christensen, K. G. Malmos and T. Vosegaard, Anal. Chem., 2019, 91, 3035-3042.

18 Y. B. Monakhova and B. W. K. Diehl, Anal. Bioanal. Chem., 2018, 410, 7891-7900.

19 J. Schiller and K. Arnold, Med. Sci. Monit., 2002, 8, MT205-22.

20 J. Folch, M. Lees and G. H. Sloane Stanley, J. Biol. Chem., 1957, 226, 497-509.

21 L. J. White, J. E. Boles, N. Allen, L. S. Alesbrook, J. M. Sutton, C. K. Hind, K. L. F. Hilton, L. R. Blackholly, R. J. Ellaby, G. T. Williams, D. P. Mulvihill and J. R. Hiscock, J. Mater. Chem. B, 2017, 8, 7620-7630.

22 B. M. Tulk, P. H. Schlesinger, S. A. Kapadia and J. C. Edwards, J. Biol. Chem., 2000, 275, 26986-26993.

23 B. M. Tulk, S. Kapadia and J. C. Edwards, Am. J. Physiol. Cell Physiol., 2002, 282, C1103-C1112.

24 L. Varela, A. C. Hendry, D. Cantoni and J. L. Ortega-Roldan, bioRxiv, 2019, 275, 638080.

25 M. Gritti, R. Würth, M. Angelini, F. Barbieri, M. Peretti, E. Pizzi, A. Pattarozzi, E. Carra, R. Sirito, A. Daga, P. M. G. Curmi, M. Mazzanti and T. Florio, Oncotarget, 2014, 5, 11252-11268.

26 M. Setti, D. Osti, C. Richichi, B. Ortensi, M. Del Bene, L. Fornasari, G. Beznoussenko, A. Mironov, G. Rappa, A. Cuomo, M. Faretta, T. Bonaldi, A. Lorico and G. Pelicci, Oncotarget, 2015, 6, 31413-31427.

27 T. Tang, X. Lang, C. Xu, X. Wang, T. Gong, Y. Yang, J. Cui, L. Bai, J. Wang, W. Jiang and R. Zhou, Nat. Commun., 2017, 8, 1-12. 